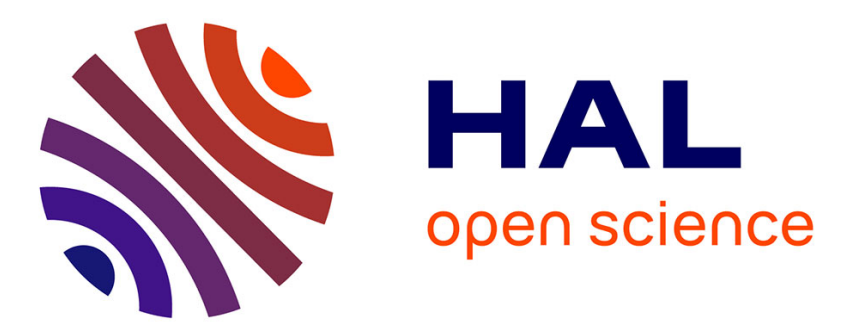

\title{
Electric vehicles performance estimation through a patterns extraction and classification methodology
}

\author{
Anthony Barré, Frédéric Suard, Mathias Gérard, Delphine Riu
}

\section{To cite this version:}

Anthony Barré, Frédéric Suard, Mathias Gérard, Delphine Riu. Electric vehicles performance estimation through a patterns extraction and classification methodology. Journal of Power Sources, 2015, 273, pp.670-679. 10.1016/j.jpowsour.2014.09.098 . hal-01071586

\section{HAL Id: hal-01071586 https://hal.science/hal-01071586}

Submitted on 12 Jul 2019

HAL is a multi-disciplinary open access archive for the deposit and dissemination of scientific research documents, whether they are published or not. The documents may come from teaching and research institutions in France or abroad, or from public or private research centers.
L'archive ouverte pluridisciplinaire HAL, est destinée au dépôt et à la diffusion de documents scientifiques de niveau recherche, publiés ou non, émanant des établissements d'enseignement et de recherche français ou étrangers, des laboratoires publics ou privés. 


\title{
Electric vehicles performance estimation through a patterns extraction and classification methodology
}

\author{
Anthony Barré ${ }^{a b}$, Frédéric Suard ${ }^{a}$, Mathias Gérard ${ }^{b}$, Delphine Riu ${ }^{\mathrm{c}}$ \\ a CEA, LIST, Data Analysis and Systems Intelligence Laboratory, 91191 Gif sur Yvette Cedex, France \\ b CEA, LITEN, 17 rue des martyrs, 38054 Grenoble Cedex 9, France \\ c G2Elab, UMR 5269, 38402 Saint Martin d'Héres, France
}

\begin{abstract}
Direct estimation of battery performance is a major challenge as ageing process is a complex phenomenon not directly measurable. In this work a new methodology is provided to estimate global battery performances under real-life electric vehicle use. Such performances are estimated through battery signals patterns extraction. These signals patterns are used to identify physical degradation behavior of batteries.

The analysis framework is composed of patterns extraction, clustering algorithms, summarizing data representation in the feature space of cluster distances and classification algorithms. This methodology is then applied on datasets, acquired from batteries used on electric vehicles, without controlled environmental conditions.

The classification algorithm accuracy is studied on the obtained real data. The results suggest that battery signals patterns analysis provides an innovative technique for online estimation of the battery performance level. A detection of dysfunctions caused by ageing is also made, only based on battery signals pattern extracted during real vehicle accelerations.
\end{abstract}

\section{Keywords}

Electric vehicles performance, Pattern analysis, Estimation, Classification, Feature clustering

\section{Introduction}

Lithium-ion (Li-ion) batteries are becoming the battery of choice in Electric Vehicles (EV) utilization. However, battery health and lifetime remain a major drawback to the use of Li-ion batteries in stringent life requirements. In EV context, accurate battery health assessment is primordial to improve the users confidence in the battery range. Indeed, it is one of the biggest obstacles to widespread acceptance of EVs. Market experts evaluated the effects of low range resources of EVs, as a significant feature for users' purchase intentions [1].

The overall performance of batteries is not constant along the vehicle life. Reduction of battery performances is caused by various internal and external mechanisms and is characterized by the capacity fade as well as an impedance augmentation [2]. For an electric vehicle (EV) utilization, the degradation of battery performances can be characterized by a diminution of the global vehicle autonomy available with a full charge [3].

Significant efforts have been achieved in order to understand the complex battery ageing process [4]. The aim for EV applications is to estimate the performance level of the battery with few measurements, in order to obtain online estimations of the battery performance level. Furthermore, ageing is a complex phenomenon, difficult to estimate with only few experiments and with online constraints. All these constraints create an intricate compromise between the model accuracy and its complexity [3].

Different methods are used to estimate the notion of battery performance level. These studies and methodologies come from many various fields such as electrochemical modeling [5], [6] or 
performance modeling [7]. The diversity and multitude of existing studies dealing with battery ageing provide a large amount of information. However, most battery ageing studies are based on direct factors dependency and have limited prediction ability. These investigations mainly rely on simulated data, under controlled conditions, which is not totally representative of a real EV use [3].

Moreover, many data-driven methodologies are focused on the battery capacityestimation [8], [9], [10]. However, most of these data-driven approaches perform well on their training data only, under specific operational experiments, inducing robustness and generalization mistakes. In real life, external conditions cannot be controlled and these learned models are subject to misestimations. Thus, an accurate way of estimating battery capacity in real-time based on real EV using data-driven algorithm still requires investigations [3].

Battery performance changes can induce modifications of its electrochemical reactions in specific conditions [11], as highlighted by incremental capacity analysis and differential voltage analysis [12], [13], [14]. Thus, the battery degradations over time lead to a modification of its behavior through the utilizations [15], [16]. These modifications can be detected with the different battery measures coming from real EV use. They can provide indications about the battery performance level. This behavior alteration is the focus of this study and will be used to estimate the battery performance level.

To explore battery performance evolution using only real-life vehicle test data is difficult and challenging. The opportunity considered here is to analyze the battery signals behavior, from data collected from real-life EV operations. In our view, revisiting battery signals behaviors using pattern extraction, analysis and classification tools may provide new insights about the relationship between these signals and the global battery performance level, being able to improve battery diagnosis and prognostics.

In this work, we propose an alternative approach by only using a data-driven methodology developed from a set of real EV uses. Such a methodology requires a large amount of training data in the development phase. In the EV context this training data requirement is very restrictive and costly. To face this problem, we investigate whether it is possible to extract relevant features from current and voltage signals collected during real EV uses, under uncontrolled conditions. A key issue explored by this paper is how battery capacity can be estimated during real EV uses, without specific requirements, based only on real use data and extracted features.

Section 2 presents the global theoretical framework and details the methods used for the extraction and classification of specific signals patterns. This will lead to an estimation of the battery capacity level, during its real uses. We study the most appropriate extracted patterns for performance classification, we also address the choice of distance metrics used for comparing battery features. In Section 3, accuracy of the methodology is evaluated for several types of classifiers, using real data. We show that an accurate analysis of the patterns morphological variation, can reveal meaningful changes in the underlying battery performances. Finally, Section 4 presents a large discussion of the proposed framework and its applications. Conclusion is given in Section 5.

\section{Proposed method}

The signals analysis framework is used in diverse application fields, showing for example its ability in classification tasks [17], [18]. The main applications of signal processing and machine learning techniques are the clustering and classification methods, which aim to separate observations into groups in the unlabeled cases [19]. Such algorithms produce a major step in data analysis and exploration. Cluster analysis is a common unsupervised learning technique for partitioning a dataset into subsets of data elements that are similar according to some distance metric [20]. Furthermore, based on clustering, groups analysis results led to producing interesting interpretations. 
The pattern analysis framework used in this study is illustrated in Fig. 1. The first step consists in a signal pattern extraction from real measured battery data, to observe the battery behavior modifications. Then, different classification methods are used to test whether the different signal morphologies represent different battery performance levels.

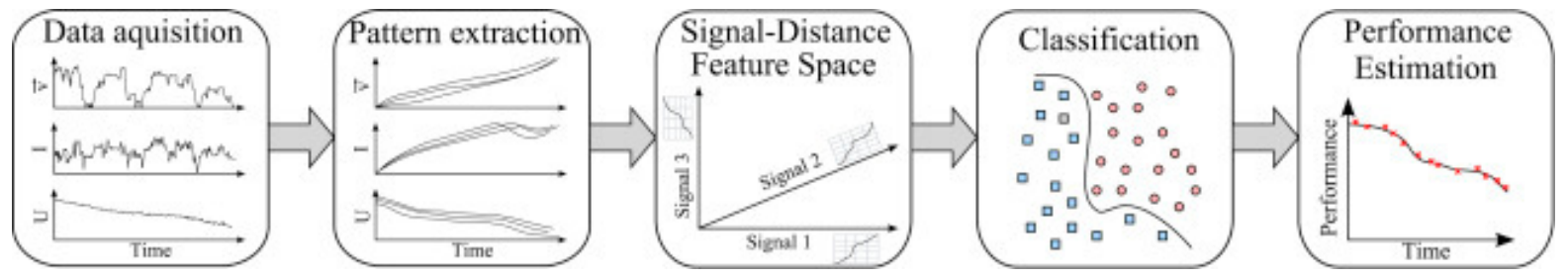

Fig. 1. Battery signals analysis framework consisting of the data acquisition, the extraction of the patterns, the formation of a new feature space, the patterns classification and the estimation of the battery performance level.

\subsection{Datasets}

Three EV instrumented $\mathrm{LiFeO}_{4}$ (LFP) batteries datasets are used in this study. In each dataset, the battery signals are acquired at $10 \mathrm{~Hz}$ frequency, during non-controlled real EV uses, thereby data representative of a large variety of conditions an EV battery can be processed. Moreover, using real data ensures compatibility of developed methodologies for embedded uses. These batteries are used on three different $\mathrm{EV}$, following a vehicle sharing concept, as several drivers alternatively take these EV for their personal travels. Thus, these experimentations are representative of the different ways an EV can be used.

Table 1 holds the main details of the battery design and their characteristics. Battery 1 and Battery 3 have a final capacity lower than Battery 2, inducing a higher degradation level and thus a more important modification of their behavior. Note that the end of life (EOL) criteria of the battery is usually defined with a capacity level equal to $80 \%$.

Table 1. Principal characteristics of the three studied batteries.

\begin{tabular}{|l|c|c|c|c|c|}
\hline & $\begin{array}{c}\text { Energy density } \\
\left(\mathrm{Wh} \mathrm{kg}^{-1}\right)\end{array}$ & $\begin{array}{c}\text { Nominal } \\
\text { voltage (V) }\end{array}$ & $\begin{array}{c}\text { Total capacity } \\
(\mathrm{Ah})\end{array}$ & $\begin{array}{c}\text { Months } \\
\text { of use }\end{array}$ & $\begin{array}{c}\text { Final capacity } \\
(\%)\end{array}$ \\
\hline Battery 1 & 80 & 400 & 75 & 59 & 87 \\
\hline Battery 2 & 75 & 128 & 95 & 45 & 91 \\
\hline Battery 3 & 72 & 134.4 & 119 & 46 & 87 \\
\hline
\end{tabular}

\subsection{Pattern extraction}

The methodology proposed in this work is based on the assumption of the battery behavior modification over time. The aim is to explore the battery response depending on its performance levels. For a similar battery request, it is possible to detect battery ageing effects based on signals shape such as current and voltage [12], [21]. Thus, both current and voltage profiles present significant behavior modifications, throughout battery life requiring investigation, characterizing their performance levels.

However, these signal modifications are low and complex to identify. To observe a battery behavior alteration, it is necessary to compare battery signals under comparable uses. For example, during an identical speed profile criterion, the battery voltage does not react the same way depending on its ageing level. 
To observe and compare those signals modifications, we have to consider only a short length signal, with a unique and common dynamic that could be used as a common reference. Hence, the signal investigation requires the definition of criteria permitting the extraction of battery patterns. Thus, duration and speed acceleration criteria define pattern extraction conditions. These extraction criteria depend on vehicle and battery characteristics and have to be consequently adapted. The impact of these duration criteria is discussed further in the following sections. Based on these criteria, the obtained patterns can allow the identification of different behaviors according to their corresponding battery performance levels.

The detection of pattern behavior alteration requires a long battery solicitation highlighting its performance level. Thus, in an EV application the ideal conditions occur during a maximum acceleration, allowing the signal patterns comparison under a common reference. Fig. 2 presents an example of pattern extraction following the criteria of an acceleration from 20 to $40 \mathrm{~km} \mathrm{~h}^{-1}$ in less than $4 \mathrm{~s}$. Thus the pattern extraction is dependent of two criteria: speed thresholds and duration bounds. Current and voltage patterns corresponding to these specific accelerations are also extracted.
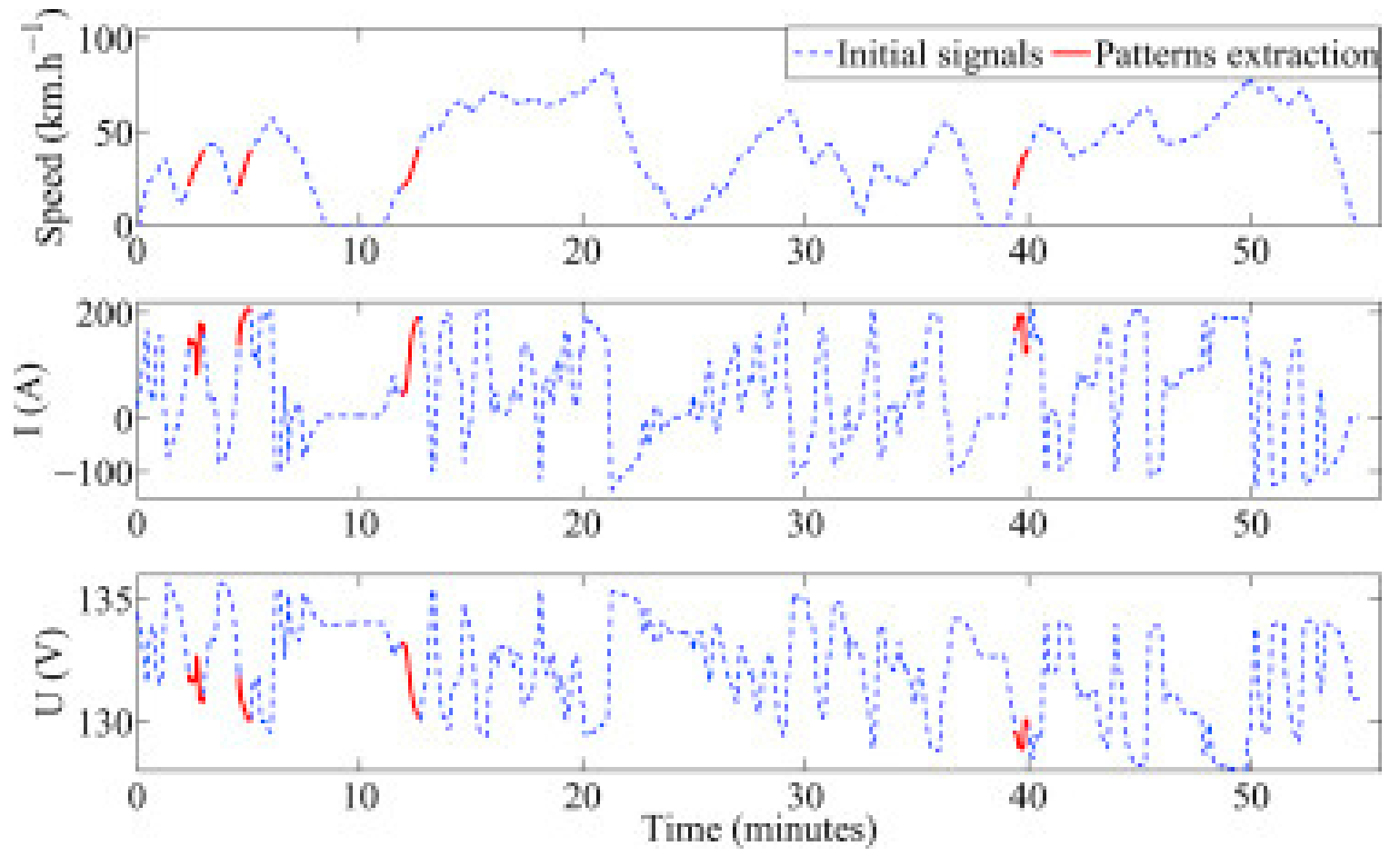

Fig. 2. Patterns extraction of an acceleration from 20 to $40 \mathrm{~km} \mathrm{~h}^{-1}$, from $E V$ battery 2 speed signal, corresponding current and voltage patterns are also extracted. 
In real-life conditions it is impossible to obtain exactly twice the same speed profile, implying a diversity among the extracted signals. Thus, the signal patterns are all different and cannot be directly compared. In order to illustrate the pattern variations depending on the battery performance levels, average signals shape are built using the Filtered Shape Averaging (FSA) method. Averaging results extracted from the 10-60 $\mathrm{km} \mathrm{h}^{-1}$ accelerations of the Battery 1, under four different performance classes are presented in Fig. 3. These classes represent four disjoint battery performance levels, sorted from the less aged period "Class 1" to the more aged battery level "Class 4". Note that it does not make sense to compare signals coming from different battery designs as their behaviors are inherently very different.
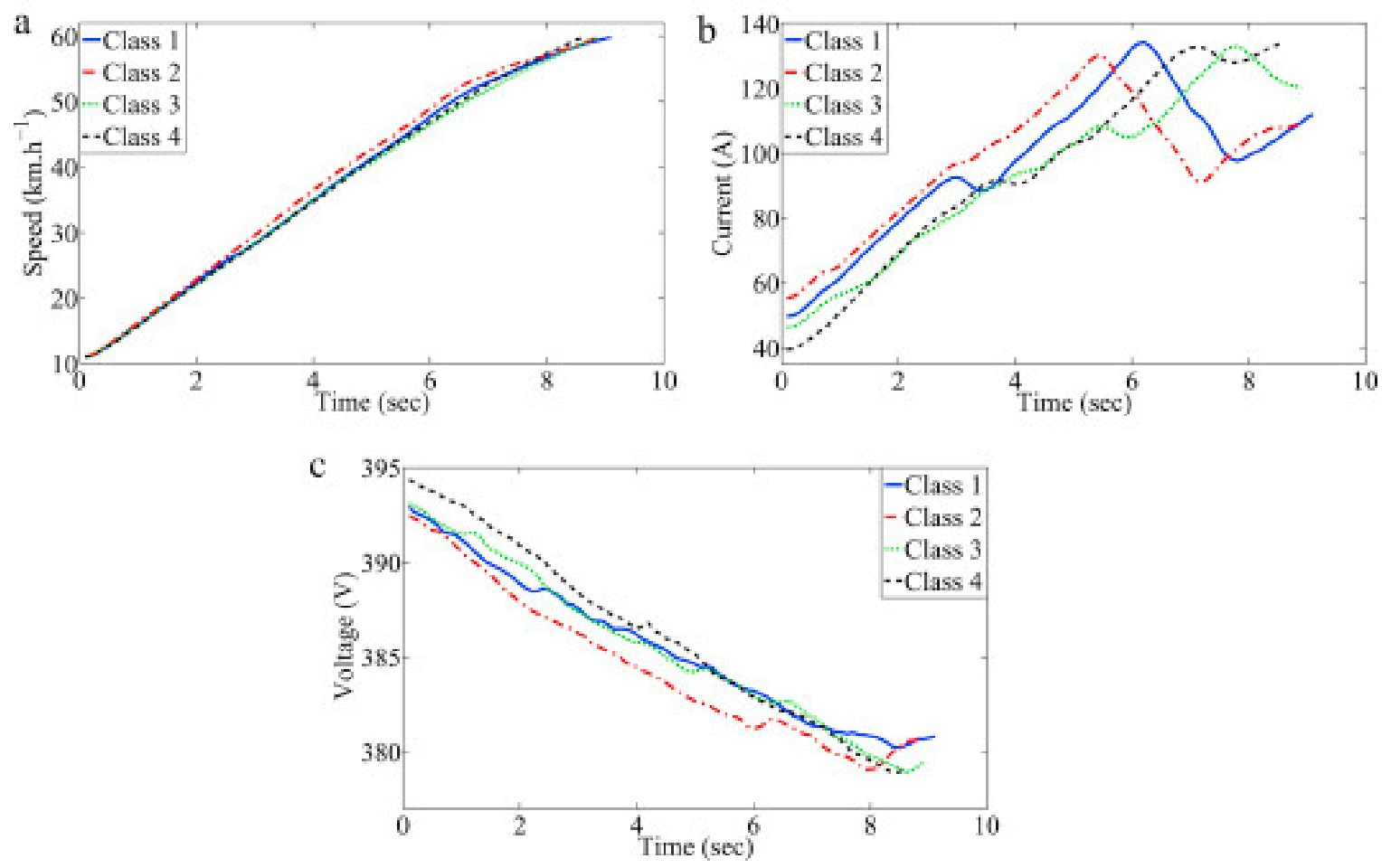

Fig. 3. (a). Speed profile - average patterns profile corresponding to a maximum acceleration under four different performance levels of battery 1 , based on $10-60 \mathrm{~km} \mathrm{~h}^{-1}$ accelerations. (b). Current profile average patterns profile corresponding to a maximum acceleration under four different performance levels of battery 1, based on $10-60 \mathrm{~km} \mathrm{~h}^{-1}$ accelerations. (c). Voltage profile - average patterns profile corresponding to a maximum acceleration under four different performance levels of battery 1, based on $10-60 \mathrm{~km} \mathrm{~h}^{-1}$ accelerations.

The same results for the battery 2 and for a shorter acceleration criteria $\left(20-40 \mathrm{~km} \mathrm{~h}^{-1}\right)$ are presented in Fig. 4. 

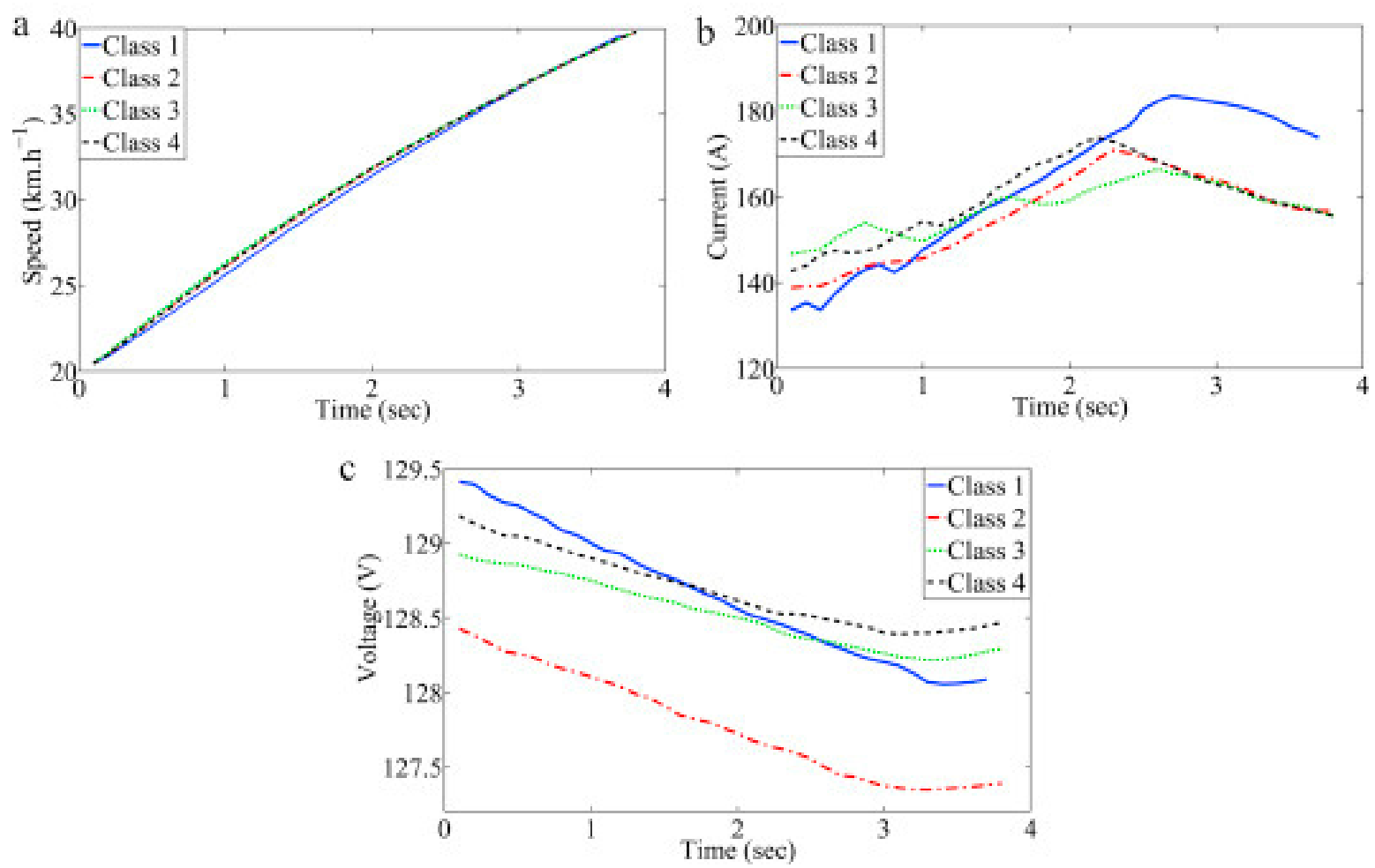

Fig. 4. (a). Speed profile - average patterns profile corresponding to a maximum acceleration under four different performance levels of battery 2 , based on $20-40 \mathrm{~km} \mathrm{~h}^{-1}$ accelerations. (b). Current profile average patterns profile corresponding to a maximum acceleration under four different performance levels of battery 2, based on $20-40 \mathrm{~km} \mathrm{~h}^{-1}$ accelerations. (c). Voltage profile - average patterns profile corresponding to a maximum acceleration under four different performance levels of battery 2 , based on $20-40 \mathrm{~km} \mathrm{~h}^{-1}$ accelerations.

Thus, Fig. 3, Fig. 4 illustrate variations of the signal morphologies for different battery performance levels. For both batteries, the extracted speed profiles are really close to each other forming good comparative samples. The corresponding current and voltage patterns are highly diverse, thus the four average patterns demonstrate a modification of the battery behavior, depending on its performance levels. Indeed, it is clearly visible in Fig. 3 and in Fig. 4 that the current and voltage behaviors are different for each of the four performance classes. For example, the current pattern of class 4 (the most aged battery level) is lower than the other ones, but on the contrary its corresponding voltage pattern is at a high level at the beginning and then decrease quicker than for the other performance classes. These patterns also present shape dissimilarities as visible in Fig. 3(b) illustrating a different shape behavior for each of the four classes. For example, average current pattern of "Class 1" decreases after $6 \mathrm{~s}$, as opposed to the growing average current pattern associated to "Class 4".

These behavior differences can be explained by a slower reaction of the battery for a same power demand over its performances level, but no direct trend can be detected. However, interpretations can be here considered but are subject to misunderstandings as the modification battery behavior is a complex problem.

The objective of the following study is to use these modifications of the battery behavior to estimate the performance level, only based on the extracted patterns. 


\subsection{Dynamic Time Warping}

In order to compare the signals pattern and quantify their similarities, we have to consider a metric adapted to this problem. Thus, beyond usual measures, the current state-of-the-art of shape similarity quantification is the Dynamic Time Warping (DTW). It permits to compare asynchronous signals of different lengths. The primary goal of DTW is to compare sequences respecting their shapes by finding an optimal alignment function stretching them. Since its introduction in the 70s, DTW has commonly been used in signals similarity problems in many fields: speech processing, signals recognition, data mining and imaging [22], [23], [24].

This method is based on the Levenshtein distance [25] and finds the optimal path between two sequences, considering temporal distortion. This optimal path produces an alignment function, along with a shape-based similarity measure. Formally, we have two sequences $X:=\left(x_{1}, \ldots, x_{N}\right)$ of length $N \in \mathbb{N}$ and $Y:=\left(y_{1}, \ldots, y_{M}\right)$ of length $M \in \mathbb{N}$. In the following we fix a feature space denoted by $\mathscr{F}$. To compare two different features $x, y \in \mathscr{F}$, one needs a local cost measure, defined by a function $c$ :

(1) $c: \mathscr{F} \times \mathscr{F} \rightarrow \mathbb{R} \geq 0$

Typically, the cost $c(x, y)$ is low if $x$ and $y$ are similar to each other, otherwise $c(x, y)$ is high. Evaluating the local cost measure $c(x, y)$ for each pair of elements of the sequences $X$ and $Y$, one obtains the cost matrix $C \in \mathbb{R}^{N \times M}$ defined by $C(i, j)=c\left(x_{i}, y_{j}\right)$. The goal is to find the alignment between $X$ and $Y$ minimizing the overall cost. A warping path is a sequence $p=\left(p_{1}, \ldots, p_{L}\right)$ with

$p_{l}=\left(n_{l}, m_{l}\right) \in[1: \mathrm{N}] \times[1: \mathrm{M}], \forall l \in[1: L]$, satisfying the following conditions:

$$
\text { (2) }\left\{\begin{array}{c}
p_{1}=(1,1) \text { and } p_{L}=(N, M) \\
n_{1} \leq \cdots \leq n_{L} \text { and } m_{1} \leq \cdots \leq m_{L} \\
p_{l+1}-p_{l} \in\{(1,0),(0,1),(1,1)\}, \quad \forall l \in[1: L-1]
\end{array}\right.
$$

A warping path $p=\left(p_{1}, \ldots, p_{L}\right)$ defines an alignment between two sequences $X$ and $Y$ by assigning the element $x_{n_{l}}$ of $X$ to the element $y_{m_{l}}$ of $Y$. The alignment conditions imply that the first elements of $X$ and $Y$ as well as their last elements are aligned to each other. The total cost $c_{p}(X, Y)$ of a warping path between $X$ and $Y$ with respect to the local cost measure $c$ is defined as:

(3) $c_{p}(X, Y)=\sum_{l=1}^{L} c\left(x_{n_{l}}, y_{m_{l}}\right)$

Examples of paths between two signals $X=\left\{x_{1}, \ldots, x_{6}\right\}$ and $Y=\left\{y_{1}, \ldots, y_{8}\right\}$ are given in Fig. 5, illustrating an admissible path following the Equation (2) (Fig. 5a) and two non admissible paths due to boundary conditions (Fig. 5b) and to step size conditions (Fig. 5c). 


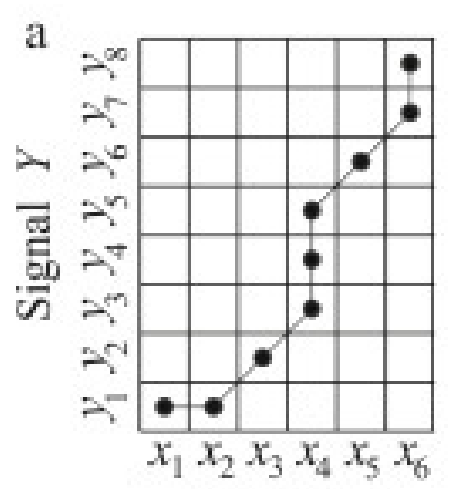

Signal $X$

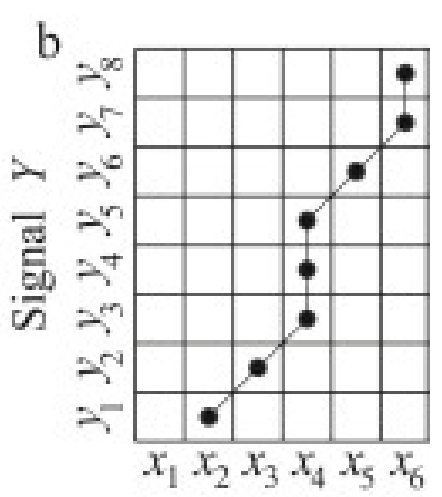

Signal $X$

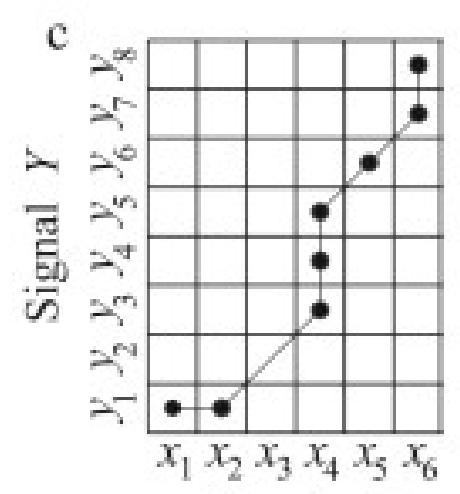

Signal $X$

Fig. 5. (a). Admissible warping path - illustration of paths of index pairs for a sequence $X=\left\{x_{1}, \ldots, x_{6}\right\}$ and a sequence $Y=\left\{y_{1}, \ldots, y_{8}\right\}$. (b). Example of a non admissible warping path due to boundary condition - illustration of paths of index pairs for a sequence $X=\left\{x_{1}, \ldots, x_{6}\right\}$ and a sequence $Y=\left\{y_{1}, \ldots, y_{8}\right\}$ (c). Example of a non admissible warping path due to step size condition - illustration of paths of index pairs for a sequence $X=\left\{x_{1}, \ldots, x_{6}\right\}$ and a sequence $Y=\left\{y_{1}, \ldots, y_{8}\right\}$.

Furthermore, an optimal warping path between $X$ and $Y$ is a warping path $p^{*}$ minimizing total cost among all possible warping paths. The DTW distance $d_{D T W}(X, Y)$ between $X$ and $Y$ is then defined as the total cost of the optimal warping path $p^{*}$ :

$$
\text { (4) } d_{D T W}(X, Y)=c_{p^{*}}(X, Y)=\min \left\{c_{p}(X, Y) \mid \forall p\right\}
$$

This DTW distance permits the comparison and the quantification of different signals shape. It is particularly adapted to battery signals evolution. Therefore, this distance measures the difference between each extracted pattern.

\subsection{Classification methods}

Based on the DTW distance applied on the extracted patterns, the aim is to estimate the battery performance level. In this section, different classification and clustering methods are presented. All of these methods require a distance permitting the comparison between observations, which is going to be the DTW distance in our application as it is adapted to the nature of the analyzed patterns.

The input of the clustering-classification framework is a dataset $B=\left\{\left(b_{1}, l_{1}\right), \ldots,\left(b_{n}, l_{n}\right)\right\}$, composed of $n$ signals pattern $b_{i}$ along with their associated performance label $l_{i}$.

The considered dataset $B$ is partitioned into a training set $B^{\text {train }}$ and a testing set $B^{\text {test }}$, permitting to build classification algorithms based on the training dataset. The following detailed classification algorithm 
provides a label estimation $\tilde{l}_{i}$ for an observation $b_{i} \in B^{\text {test }}$. Since the class labels represent the battery performance level, the classification accuracy CA on a method is defined as the percentage of data elements classified in their actual label $l_{i}$ :

$$
\text { (5) } C A=\frac{\left|\left\{b_{i} \in B^{\text {test }} \mid \tilde{l}_{i}=l_{i}\right\}\right|}{\left|B^{\text {test }}\right|}
$$

\subsection{1. $K$-Nearest Neighbors}

The $K$-Nearest Neighbors $(K-\mathrm{NN})$ approach has been widely investigated and has shown to be a powerful non-parametric technique in classification problems. This algorithm is computationally simple and commonly used as a reference, thus a new object is assigned to the majority class voted among its $K$ nearest neighbors in the training set [26]. Formally, given a labeled training set $B^{\text {train }}$ and a test pattern $b_{i} \subset B^{\text {test }}, K$-NN classifies by choosing $K$ train data elements $\left\{b_{1}, \ldots, b_{K}\right\} \subset B^{\text {train }}$ that are the $K$ closest neighbors of $b_{i}$ under the used metric. Then, $b_{i}$ will be classified as the statistical mode from the labels of the $K$ nearest neighbors $\left\{l_{1}, \ldots, l_{K}\right\}$. Note that the parameter $K$ is a positive integer strongly influencing the algorithm accuracy.

\subsection{2. $K$-Means}

The $K$-Means algorithm is one of the most popular and widely used as a clustering method. This algorithm follows a local optimization strategy. It starts with an initial partition of the $n$ observations of $B^{\text {train }}$ into $K$ clusters, and then uses an iterative process to optimize the clusters quality.

The aim is to group $n$ observations into $K$ separate clusters $\left\{C_{1}, \ldots, C_{K}\right\}$, so that the within-cluster sum of squares is minimized. This distortion $E$ is measured by $E=\sum_{i=1}^{n}\left(\min _{k=1, \ldots, K} D\left(b_{i}, C_{k}\right)\right)$, with $D$ the DTW distance measure. The distance between an element $b_{i}$ and a cluster $C_{k}$ is defined as the distance between $b_{i}$ and the center of this cluster $c_{k}^{(t)}$ at the step $t$. This center of a cluster is built according to the Filtered Shape Averaging (FSA) method, producing an average signal representative of the class behavior, using the DTW optimal path with an iterative process. Thus, this methodology is dependent on the choice of initial positions of cluster centers $\left(c_{1}^{(0)}, \ldots, c_{K}^{(0)}\right)$. In this study these initial positions are randomly chosen among the observations. At the end of the iterative procedure, all observations of the training set $B^{\text {train }}$ are assigned to a single cluster.

With the formation of the $K$ clusters, a new observation $b_{i} \in B^{\text {test }}$ is associated to the nearest cluster $C_{j}$. Thus, the label associated to the pattern $b_{i}$ is the statistical mode from the labels of this cluster $C_{j}$.

\subsubsection{Hierarchical}

Hierarchical clustering is applied to the $n$ training patterns of $B^{\text {train }}$ to obtain a partition of this dataset into disjoint clusters, in a way that data elements within the same cluster share a similarity according to the chosen DTW distance. This clustering is done following an agglomerative hierarchical clustering procedure [27]that initially partitions a set of $n$ data elements into $K$ distinct clusters, each containing one data element, and then iteratively merges the two most similar clusters, until the entire dataset is aggregated into a unique cluster. The created hierarchical tree can next be pruned so that the number of clusters $K$ is obtained. 
Thus, data elements below each cut are assigned to a single cluster, creating the output data partitioning to clusters $\left\{c_{1}, \ldots, c_{K}\right\}$. The algorithm requires a cluster similarity criterion for choosing the next two clusters to be merged. We have simply used the average similarity between all the centers of both clusters.

Following the same classification process as the $K$-Means algorithm, a new observation is associated to the label of its nearest cluster.

\subsubsection{Support Vector Machine}

The theoretical foundations of Support Vector Machine (SVM) were developed by Vapnik [28] the method has been highly explored during recent years. The SVM method is applied for classification in various domains providing excellent results [29], [30], [31], [32]. Using the set of training patterns $B^{\text {train }}$, and their corresponding class identifiers; it assigns the correct class to a newly presented object. For the task of learning, a measure of similarity between the objects of $B^{\text {train }}$ is necessary, so that classifiers are created separating the data space into separate areas. A reasonable measure of similarity is of the form $k: \mathrm{B}^{\text {train }} \times \mathrm{B}^{\text {train }} \rightarrow \mathbb{R}$, where $k$ is called a kernel. In our application, the chosen kernel $k$ is the DTW Kernel (DTWK) [33], permitting the use of the DTW metric in the SVM method.

Note that the SVM classification is initially adapted to a two-class problem. In order to adapt the SVM to a multi-class we reduce the single multiclass problem into multiple binary classification problems [34].

\section{Results on real data}

The described framework is applied separately on each battery dataset. Several extraction criteria are studied, permitting the comparison of the results depending on the patterns. Thus, the global methodology is applied on two types of speed profile, one considering an acceleration from 20 to $40 \mathrm{~km} \mathrm{~h}^{-1}$, and the other from 10 to $60 \mathrm{~km} \mathrm{~h}^{-1}$. Notice that the longest acceleration contains more information. However, longer acceleration profiles require larger datasets to obtain enough patterns for the methodology process. These extracted patterns are then used in the global algorithm and the results are explored.

\subsection{Clustering}

The first step consists in the exploration of the patterns clustering results. Hierarchical clustering and $K$ Means methodologies can be applied to the extracted patterns described above. Cluster analysis is able to identify distinct patterns morphologies. Fig. 6 presents for example a clustering based on current patterns extracted during specific accelerations, with the average current patterns of each cluster resulting of the FSA methodology. These results illustrate the diversity of the battery current shape between the various 8 clusters. For example, cluster 8 presents decreasing currents after $7 \mathrm{~s}$, as opposed to cluster 4 containing constantly growing current patterns. Note that the speed profile corresponding to the current patterns, as previously explained, does not present significant differences due to the pattern extraction mode. This result permits to demonstrate the variability of the current patterns behavior. 

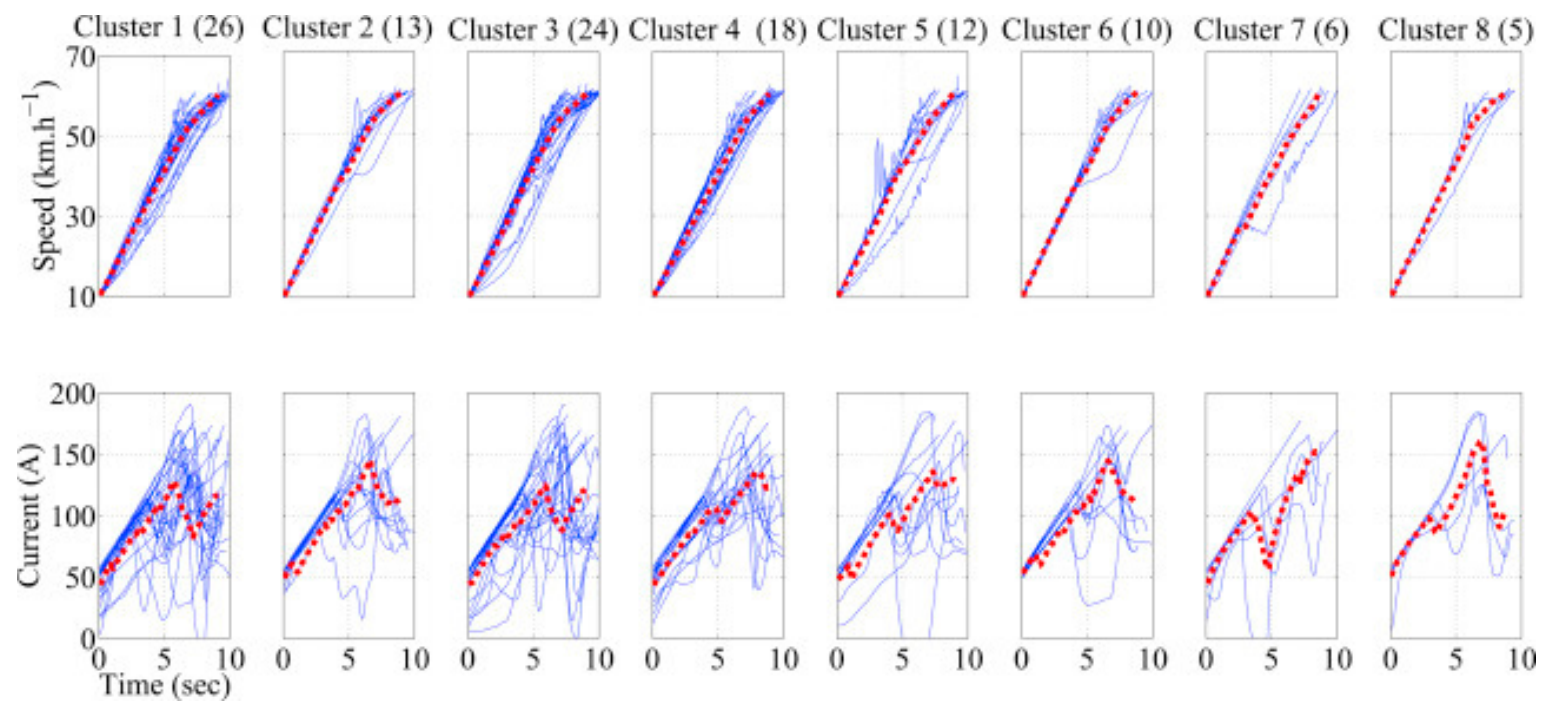

Fig. 6. Clustering result of the $K$-Means method for $10-60 \mathrm{~km} \mathrm{~h}^{-1}$ accelerations of battery 1 - the number of patterns in each cluster are in parenthesis, in red is presented an average current pattern of each cluster, obtained by FSA. (For interpretation of the references to color in this figure legend, the reader is referred to the web version of this article.)

As the data are provided from real-life tests, these differences can be caused by external conditions such as temperature, State Of Charge (SOC) battery level. Indeed, a cluster can for example be composed of pattern measured during summer only, whatever the battery performance level.

To demonstrate the independence between the cluster composition and the external conditions, Fig. 7(a) and (b) presents the observations distribution over temperature and SOC conditions, with their associated cluster. Fig. 7 illustrates the homogeneous distribution of temperature and SOC over clusters. Thus, given a temperature or a SOC it is impossible to predict its associated cluster. To quantify these relationships, the absolute correlation coefficient between the temperature and the selected cluster mainly lower than 0.1 , as well as the correlation between SOC and clusters. These results clearly illustrate the global variability of external conditions in all clusters, and the independence between a cluster composition and their corresponding external conditions. We can conclude that external conditions have no influence on the results.
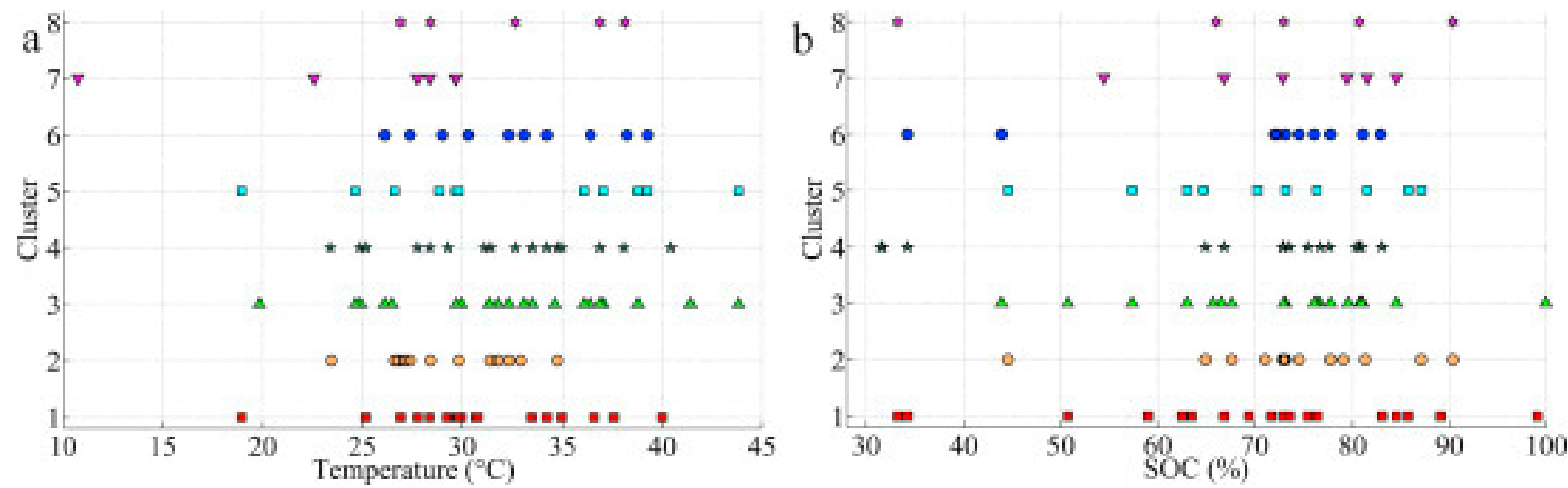

Fig. 7. (a). Temperature repartition of the clusters - distribution of the external conditions of each observation, along with their selected cluster based on a $K$-Means clustering with the battery 1 . (b). SOC repartition of the clusters - distribution of the external conditions of each observation, along with their selected cluster based on a $K$-Means clustering with the battery 1 .

Based on these cluster investigations the battery behavior on a small acceleration part permits the identification of patterns shape differences according to its performance level regardless of the external conditions. 
It is also considered to use this approach to identify behavior characteristics of a battery fault. This fault detection can be made by a simple outlier identification with the use of this clustering work. Thus, a battery default will be clearly visible as the corresponding signal patterns will be isolated in a unique cluster. This methodology consequently permits a rapid diagnosis of the global battery behavior to detect abrupt changes of battery behavior representative of a malfunction.

\subsection{Classification}

The clustering investigation induces a good separation of the patterns studied, according to their respective level of performance. The idea is now to use these modifications of the battery behavior to predict its level of performance, only based on a pattern extracted from a single acceleration. The four classification methods described in Section 2.4 ( $K$-NN, $K$-Means, Hierarchical and SVM) are used and their results are compared based on the different datasets.

Three datasets coming from three different batteries are explored, as explained in Section 2.1. Each dataset corresponds to a real EV use. In order to compare the classification methods accuracy, the methodology is applied to different sets of extracted patterns corresponding to a $20-40 \mathrm{~km} \mathrm{~h}^{-1}$ or to a $10-60 \mathrm{~km} \mathrm{~h}^{-1}$ acceleration. Table 2 presents the number of extracted patterns by battery during their entire experimentations, for two different acceleration profiles criteria. For example, on Battery 1, 143 patterns satisfy the extraction criteria of a $10-60 \mathrm{~km} \mathrm{~h}^{-1}$ acceleration during a specific duration, depending on the vehicle characteristics, defined by experts. Note that the number of extracted patterns is highly dependent of the battery uses, explaining the number of patterns corresponding to 20 $40 \mathrm{~km} \mathrm{~h}^{-1}$ accelerations in Battery experiments. Thus, extraction process provides a large amount of patterns corresponding to an identical speed profile.

Table 2. Number of extracted patterns for each battery with two different acceleration criteria.

\begin{tabular}{|l|c|c|}
\hline & $10-60 \mathrm{~km} \mathrm{~h}^{-1}$ & $20-40 \mathrm{~km} \mathrm{~h}^{-1}$ \\
\hline Battery 1 & 143 & 5192 \\
\hline Battery 2 & 139 & 662 \\
\hline Battery 3 & 249 & 676 \\
\hline
\end{tabular}

Each dataset $B=\left\{\left(b_{1}, l_{1}\right), \ldots,\left(b_{n}, l_{n}\right)\right\}$ is composed with different extracted patterns $b_{i}$, associated to a class of battery performance $l_{i}$. These datasets $B$ are then separated into a training part $B^{\text {train }}$ consisting of $75 \%$ of the entire dataset $B$, and into a test set $B^{\text {test }}$ composed with the other $25 \%$ of the dataset $B$.

As explained in Section 2.4, each classification method builds a model based on a training part $B^{\text {train }}$ of this dataset. The learned model is then applied to the test patterns $b_{i} \in B^{\text {test }}$, and the classification accuracy CA is the proportion of well classified test elements. Note that for each method, the parameters are defined following a cross validation step using the training dataset. Hence, the parameters are automatically defined during the preprocessing step.

In this section we apply classification methodologies to extracted voltage and current patterns, and to their corresponding power pattern.

\subsection{Two classes}

Consider here a binary classification for extracted patterns. For each battery, the two disjoint classes correspond to a performance class level. Thus, one class contains the observations associated with the first quarter of the performance level and the other class holds a last quarter of battery performances. It 
means that the first quarter of the battery life is opposed to the last quarter, each of them composing a specific performance class. Thus, for a battery, this classification uses only half of its total extracted patterns. Considering a test pattern, its corresponding battery performance class is estimated by each of the presented classification methods. Table 3 presents a comparison of the accuracies of each classification method, for the three batteries and for two different acceleration criteria.

Table 3. Classification accuracy of a two-class classification of the battery performance level for each pattern $(\%)$ - in bold the best accuracies $(>75 \%)$.

\begin{tabular}{|c|c|c|c|c|c|c|c|c|c|}
\hline & \multicolumn{3}{|c|}{ Bat. 1} & \multicolumn{3}{|c|}{ Bat. 2} & \multicolumn{3}{|c|}{ Bat. 3} \\
\hline & I & $\mathrm{U}$ & $\mathrm{P}$ & I & $\mathrm{U}$ & $\mathrm{P}$ & I & $\mathrm{U}$ & $\mathrm{P}$ \\
\hline \multicolumn{10}{|l|}{$20-40 \mathrm{~km} \mathrm{~h}^{-1}$} \\
\hline$K-\mathrm{NN}$ & 72 & 77 & 74 & 65 & 60 & 56 & 69 & 89 & 69 \\
\hline$K$-Means & 67 & 64 & 66 & 63 & 62 & 60 & 69 & 81 & 69 \\
\hline Hierarchical & 61 & 63 & 60 & 56 & 62 & 60 & 67 & 85 & 69 \\
\hline SVM & 69 & 70 & 71 & 64 & 62 & 61 & 67 & 86 & 67 \\
\hline \multicolumn{10}{|l|}{$10-60 \mathrm{~km} \mathrm{~h}^{-1}$} \\
\hline$K-\mathrm{NN}$ & 79 & 59 & 69 & 66 & 80 & 69 & 80 & 90 & 78 \\
\hline$K$-Means & 79 & 72 & 76 & 60 & 71 & 71 & 71 & 75 & 71 \\
\hline Hierarchical & 60 & 62 & 59 & 54 & 69 & 69 & 69 & 77 & 71 \\
\hline SVM & 72 & 72 & 69 & 63 & 69 & 63 & 76 & 80 & 75 \\
\hline
\end{tabular}

The presented results of Table 3 clearly demonstrate the ability of the methodology to perform with a good accuracy in the battery class of performance identification. Indeed, the best accuracy is obtained with the $10-60 \mathrm{~km} \mathrm{~h}^{-1}$. This can be explained because as the signal gets larger it contains more and more information on the battery behavior. Thus, the best performance is obtained with Battery 3 over 10$60 \mathrm{~km} \mathrm{~h}^{-1}$ accelerations with the $K$-NN method, based only a training dataset composed of less than 200 patterns.

These results also point out the $K$-NN and the SVM as the most accurate methods. These methods yield a correct battery performance class for a new signal pattern of around $75 \%$, depending on the patterns lengths and the dataset size. Note that the classification accuracy is up to $90 \%$ for the $K$-NN method based on voltage patterns extracted from $10-60 \mathrm{~km} \mathrm{~h}^{-1}$. This result is presented is Fig. 8, illustrating the methodology accuracy. Compared to a random process giving a $50 \%$ accuracy by assigning to each pattern one of the two classes randomly, the presented methodology demonstrates its ability to estimate battery performance level. 


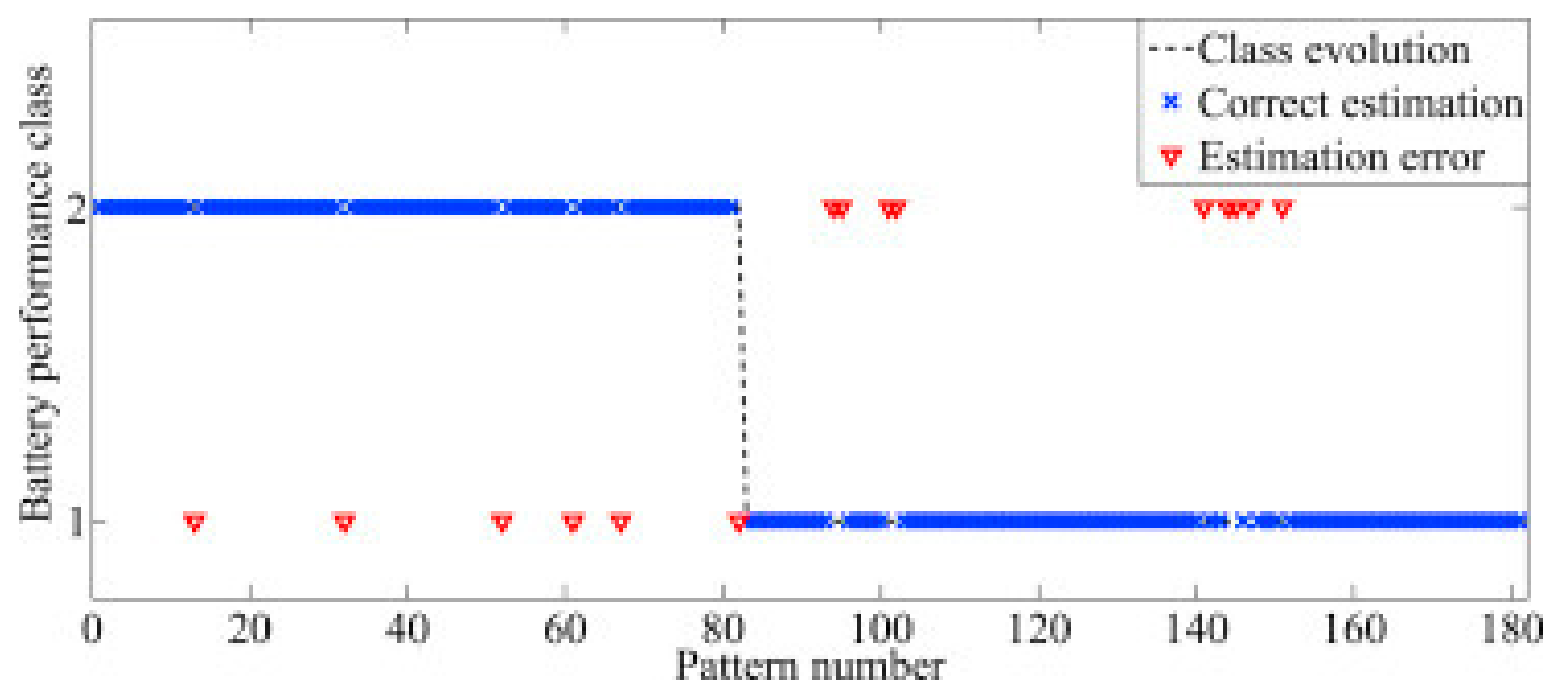

Fig. 8. Prediction distribution of the performance class by the $K$-NN method, based on voltage patterns of $10-60 \mathrm{~km} \mathrm{~h}^{-1}$ acceleration of battery 3 .

Note that the performances obtained from Battery 2 are lower than other batteries, which is explained by the fact that this battery has a higher final capacity than the two others batteries, providing fewer patterns due to the low utilizations of this Battery 2 and consequently a reduced set of training data $B^{\text {train }}$. Moreover, this Battery 2 was less aged than the others batteries, inducing a smaller modifications of its behavior. The signals alterations are more complex to detect than for a battery with a long utilization life. Consequently its behavior modifications are less sensible and the patterns alterations are more difficult to detect.

Using the $K$-NN approach applied to Battery 3 , we propose a sensitivity study of the pattern extraction criteria. We consider the fix speed accelerations thresholds at $10-60 \mathrm{~km}^{-1}$, and we use diverse duration bounds to extract corresponding voltage patterns. Thus for each acceleration duration bounds, the $K$ NN methodology creates a model based on the extracted patterns. All of these tests allow the production of an accuracy level of the formed model. Fig. 9 illustrates the model accuracy evolution for different acceleration duration bounds.

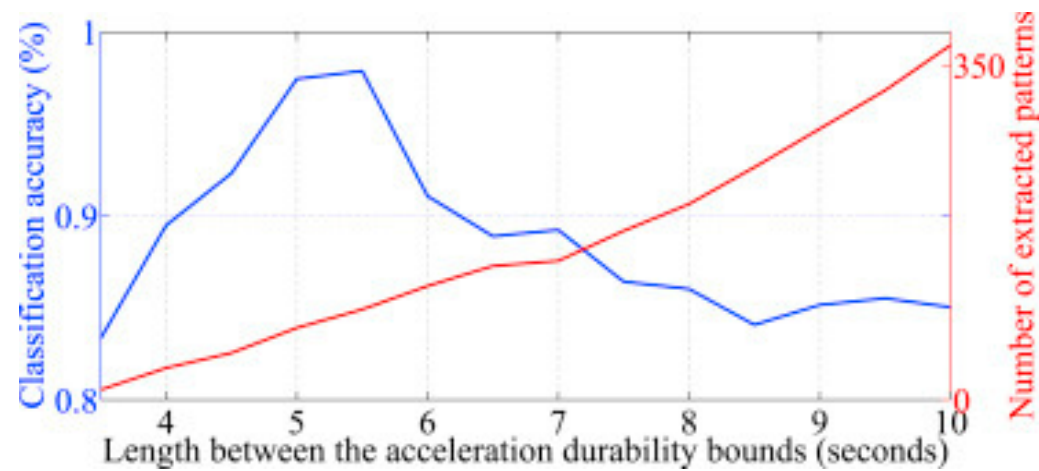

Fig. 9. Evolution of the two-classes $K$-NN accuracy from patterns extracted from 10 $60 \mathrm{~km} \mathrm{~h}^{-1}$ accelerations, of battery 3 , with different duration bounds.

Fig. 9 illustrates the compromise in the choice of extraction criteria. We can see the possibility to improve the accuracy presented in Table 3 from $90 \%$ to a $98 \%$ accuracy, by considering reduced acceleration duration bounds. This bound definition is an important part of the pattern extraction methodology as the extraction criteria are currently defined a-priori from expert advices. 


\subsection{Four classes}

With a good global accuracy in a two-classes classification, the aim is to use the same methodology to predict with a greater precision the battery performance level. Thus, Table 4 presents the classification rates of 4-class classifications. The interpretation of these results is similar to the conclusion made from the 2-class results. Indeed, the best classification performances are obtained with the $K$-NN method, for the $10-60 \mathrm{~km} \mathrm{~h}^{-1}$ accelerations. Note that a random classification gives here an accuracy of $25 \%$, thereby affirming that the global methodology permits the estimation of the battery performance level with a good success rate. It is important to observe the low results obtained by the SVM methods, opposed to the performances of the previous 2-class approach. This comment is here explained by the definition of the used kernel in the SVM process. Indeed the defined DTW Kernel (DTWK) is not positive definite in theory, but is commonly p.d. in practical experiments. However, the results presented in Table 4 demonstrate the limitation of this kernel's utilization.

Table 4. Classification accuracy of a four-class classification of the battery level of performance for each datasets (\%) - in bold the best accuracies (>50\%).

\begin{tabular}{|c|c|c|c|c|c|c|c|c|c|}
\hline & \multicolumn{3}{|c|}{ Bat. 1} & \multicolumn{3}{|c|}{ Bat. 2} & \multicolumn{3}{|c|}{ Bat. 3} \\
\hline & I & U & $\mathrm{P}$ & I & U & $\mathrm{P}$ & I & U & $\mathrm{P}$ \\
\hline \multicolumn{10}{|l|}{$20-40 \mathrm{~km} \mathrm{~h}^{-1}$} \\
\hline$K-\mathrm{NN}$ & 47 & 42 & 48 & 29 & 35 & 33 & 41 & 62 & 41 \\
\hline$K$-Means & 40 & 37 & 41 & 35 & 42 & 33 & 49 & 69 & 42 \\
\hline Hierarchical & 31 & 30 & 32 & 30 & 31 & 31 & 45 & 58 & 44 \\
\hline SVM & 37 & 36 & 37 & 33 & 32 & 30 & 43 & 55 & 40 \\
\hline \multicolumn{10}{|l|}{$10-60 \mathrm{~km} \mathrm{~h}^{-1}$} \\
\hline$K-\mathrm{NN}$ & 48 & 38 & 48 & 46 & 49 & 43 & 51 & 67 & 48 \\
\hline$K$-Means & 48 & 48 & 52 & 49 & 46 & 40 & 44 & 55 & 42 \\
\hline Hierarchical & 45 & 31 & 35 & 28 & 40 & 40 & 41 & 57 & 34 \\
\hline SVM & 48 & 34 & 38 & 37 & 46 & 34 & 32 & 42 & 33 \\
\hline
\end{tabular}

The results of a 4-class $K$-NN, based on voltage patterns from the $10-60 \mathrm{~km} \mathrm{~h}^{-1}$ of Battery 3, are presented in Fig. 10. This Fig. 10 illustrates the ability of the proposed methodology to estimate most of times the correct battery performance class, only based here on the extracted voltage pattern. For example, the observations corresponding to the battery performance class 1 are strongly well classified. However, we can observe mispredictions, especially between close classes, but rarely for remote classes. 


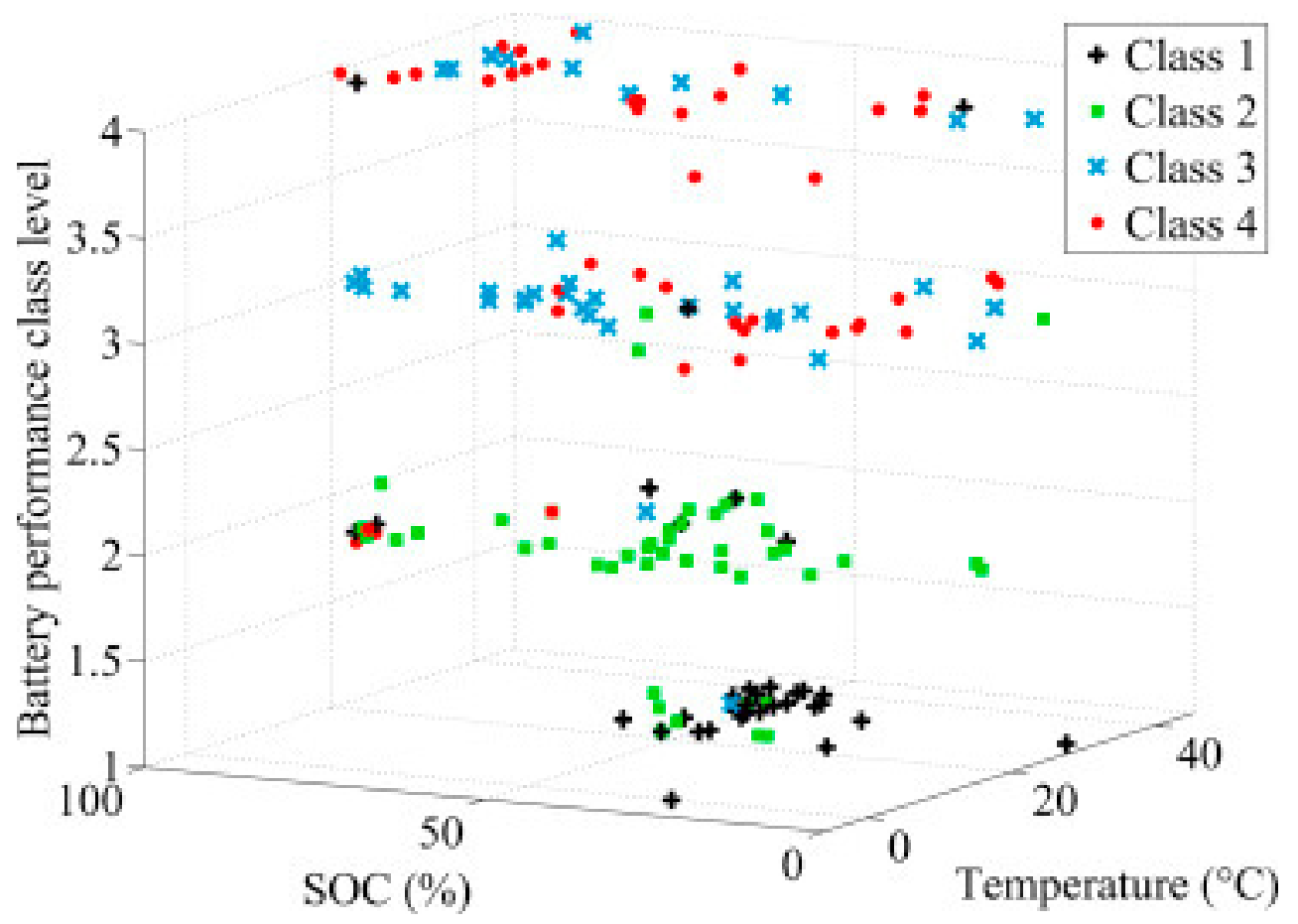

Fig. 10. Prediction repartition of the performance class by the $K$-NN method with battery 3 , depending on the temperature and SOC values.

This representation demonstrates the independence between the prediction made and the unused variables. This independence of clustering on battery temperature and SOC is illustrated by the homogeneous distribution of each of these conditions in all four composed classes. For example, the temperature distribution contained into the class 2 contains sample associated with low values $\left(\sim 0{ }^{\circ} \mathrm{C}\right)$ as well as high temperatures $\left(\sim 40^{\circ} \mathrm{C}\right)$. That is to say that the built model equally performs for all values of the State Of Charge (SOC) and of the internal battery temperature.

Thus, the presented results demonstrate the good precision of the methodology as a unique voltage pattern permits to estimate the battery performance level with an interesting accuracy. Furthermore it is interesting to observe the different accuracies depending on the used signal. Indeed, the voltage patterns provide the best results, inducing that the battery behavior modifications are more detectable inside the voltage signals than in current signals. On the contrary, the power patterns lead to lower accuracy rate. This point can be explained as the power is defined as the product between current and voltage. Thus both behaviors of current and voltage can be attenuated or altered by this product. For the best estimation precision, the presented results tend to advocate the choice of voltage patterns in the global methodology.

In order to explore the methodology performances on smaller performances classes, Fig. 11 presents the results of the $K$-NN methods on an 8-class classification, only based on voltage patterns from 20 $40 \mathrm{~km} \mathrm{~h}^{-1}$. The choice of these acceleration criteria is made to obtain enough training patterns to perform in a 8-classes approach. 


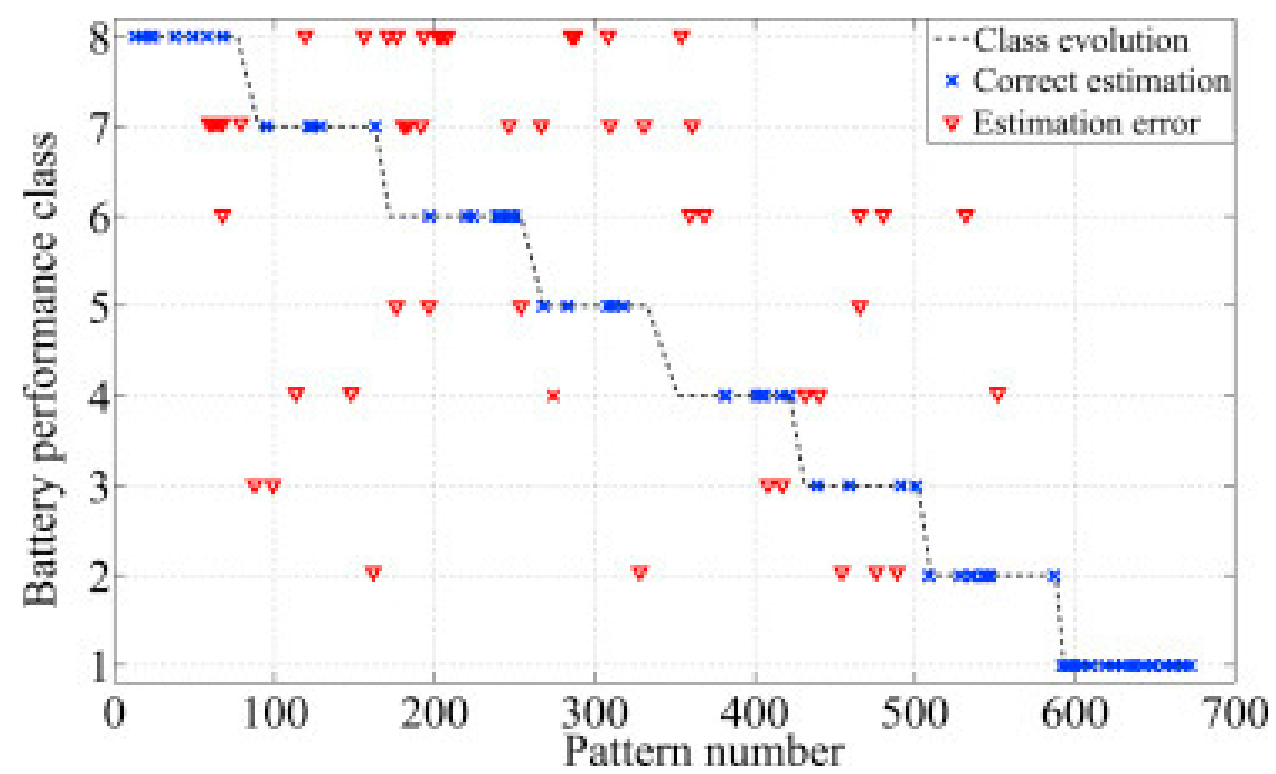

Fig. 11. Estimation repartition of the 8-classes $K$-NN method, based on voltage patterns of 20 $40 \mathrm{~km} \mathrm{~h}^{-1}$ acceleration of battery 3 - with a $55 \%$ accuracy.

This result demonstrates the good detection of battery behavior modifications depending on its performance level, only based on patterns of voltage extracted on a simple acceleration. Indeed, most class estimations are correct or are a neighbor of the real class. For example, in Fig. 11 misclassed Class 8 patterns are associated to Class 7 , corresponding to the nearest performance class of Class 8 . The difference between the battery performance classes is here less than $2 \%$ of the battery capacity, inducing the good identification of the battery behavior alteration.

\section{Discussion}

The proposed statistical approach provides an innovative framework to use patterns from battery signals. This study does not consider physical hypothesis allowing an adaptation of the global process with any battery technology and perform in real uses without specifications, demonstrating the powerful of signal patterns investigation.

Simple patterns extraction and their analysis, presented in Section 2.2 permit the identification of a battery behavior modification over different performance levels (Fig. 3, Fig. 4). Then, deeper signal patterns investigation, introduced in Section 2.4, demonstrates the ability of the patterns extraction to highlight the battery behavior, only based on the signals coming from real accelerations. Indeed, the results of Section 3 confirm the interest of this methodology in the estimation of the battery performance level, and for fault detection.

The four-classes classification results presented in Table 4 illustrate the good accuracy of the methodology to detect battery behavior over the performance level, only based on the battery signal patterns measured during vehicle uses, without requirement or specification. The obtained results present diverse accuracy for the selected patterns extraction mode, thus it is of paramount importance to define optimal patterns extraction criteria, among all accelerations profiles, illustrated by Fig. 9. This study highlights the importance of the pattern extraction criteria definition, as they directly impact the accuracy of the developed methodology. In this presented experiment all these extractions criteria are preliminary defined manually, however Fig. 9 demonstrates the significance of exploring other definition modes to detect optimal criteria.

Table 2 details the number of extracted patterns for two different acceleration profiles, illustrating the diversity of extracted patterns according to speeds considered. Indeed, a $10-60 \mathrm{~km} \mathrm{~h}^{-1}$ acceleration pattern is harder to obtain during real EV uses than a $20-40 \mathrm{~km} \mathrm{~h}^{-1}$ speed profile. 
Thereby, the presented experiments provide result illustrations with patterns extracted from the 10 $60 \mathrm{~km} \mathrm{~h}^{-1}$ accelerations. Indeed, longer patterns contain more battery behavior information and thus permit a better identification of the battery performance. This compromise between frequency and accuracy of the estimations has to be selected in accordance with the applications constraints. Consequently, better estimation results are obtained with Battery 3 containing the most extracted patterns, with $10-60 \mathrm{~km} \mathrm{~h}^{-1}$ accelerations criteria. Among the detailed classification methods, the $K$ $\mathrm{NN}$ approach is associated to the best estimations accuracies inducing a preference for the application of this method. However, the SVM algorithm permits to obtain high accuracies in a two-class context as opposed to a four-class study. These SVM limitations are here explained by the used kernel drawbacks as the considered DTWK is not positive definite, presenting difficulties in its applications.

The next step of this framework is to explore the selection of the acceleration criterion to have the best compromise between model accuracy and number of patterns, with an automatic process. The SVM results also induce a possible way to improve algorithm performances, by the use of other DTW based kernels. Moreover, the natural extension of this study is to adapt these methodologies to obtain linear estimations. This would permit, during electric uses, an online precise continuous estimation of the battery performance instead of a level class. It would also be interesting to test this methodology with a larger fleet to observe the different battery behaviors and exploit further various data to obtain a better global accuracy.

\section{Conclusion}

The aim of this study is to use an innovative methodology built from a real use signals dataset, permitting an online estimation of the battery performance, without requirement or specification. The dataset is formed from real vehicle uses and is used to create model. A preprocessing step permits to extract acceleration patterns in these measured signals, under specific extractions criteria. Once these battery signals patterns extracted, the battery behavior is highlighted to be altered by the performances levels. These patterns are then used as a training dataset in the battery performance classification approach.

By carefully exploring the signals alterations, we have illustrated an innovative methodology to demonstrate that simple pattern recognition and classification techniques permit to diagnose battery performance. This pattern analysis methodology is also able to detect battery fault detection and permits the identification of an abnormal battery behavior.

The presented methodology demonstrates the classification of the battery performances in different class levels with an interesting confidence level. We continue to find new methodologies and new pattern recognition algorithms to improve presented accuracy results.

However, the final aim is to obtain a continuous estimation of the battery performance in real-time. Thus, the natural objective is to extend this pattern signals investigation methodology to accurately quantify a battery performance. The signals pattern analysis and the relationship to battery performance degradation are the main focus of our current works. Our ultimate goal is to extend this methodology and these results to accurately estimate, online, a battery performance value, only from the signals coming from a real-life use.

\section{Acknowledgments}

The authors are grateful to Gino Paganelli and Olivier Joye of Michelin for their contribution on CEA pack integration into Michelin vehicle named WILL and for performing intensive tests on Michelin circuit. This work was supported by French agency ADEME into the "AMI - Demonstration project for new energy technologies". 


\section{References}

[1] A. Peters, E. Dütschke, J. Environ. Policy Plan. 1-19, arXiv: http://www.tandfonline.com/doi/pdf/10.1080/1523908X.2013.879037, http://dx.doi.org/10.108 0/1523908X.2013.879037,

URL: http://www.tandfonline.com/doi/abs/10.1080/1523908X.2013.879037.

[2] M. Kassem, J. Bernard, R. Revel, S. Pélissier, F. Duclaud, C. Delacourt J. Power Sources, 208 (2012), pp. 296-305, 10.1016/j.jpowsour.2012.02.068 URL: http://www.sciencedirect.com/science/article/pii/S0378775312004284

[3] A. Barré, B. Deguilhem, S. Grolleau, M. Gérard, F. Suard, D. Riu J. Power Sources, 241 (2013), pp. 680-689, 10.1016/j.jpowsour.2013.05.040 URL: http://www.sciencedirect.com/science/article/pii/S0378775313008185

[4] J. Remmlinger, M. Buchholz, M. Meiler, P. Bernreuter, K. Dietmayer J. Power Sources, 196 (12) (2011), pp. 5357-5363, 10.1016/j.jpowsour.2010.08.035

Selected papers presented at the 12th Ulm ElectroChemical Talks (UECT): 2015 Technologies on Batteries and Fuel Cells, URL:

http://www.sciencedirect.com/science/article/pii/S0378775310013534

[5] B. Stiaszny, J.C. Ziegler, E.E. Krauß, J.P. Schmidt, E. Ivers-Tiffée J. Power Sources, 251 (2014), pp. 439-450, 10.1016/j.jpowsour.2013.11.080 URL: $\underline{\text { http://www.sciencedirect.com/science/article/pii/S0378775313019150 }}$

[6] T. Waldmann, M. Wilka, M. Kasper, M. Fleischhammer, M. Wohlfahrt-Mehrens J. Power Sources, 262 (2014), pp. 129-135, 10.1016/j.jpowsour.2014.03.112 URL: http://www.sciencedirect.com/science/article/pii/S0378775314004352

[7] A. Marongiu, M. Roscher, D.U. Sauer Appl. Energy (2014), 10.1016/j.apenergy.2014.06.063

URL: http://www.sciencedirect.com/science/article/pii/S030626191400645X

[8] B. Saha, K. Goebel

Aerospace Conference, IEEE (2008), pp. 1-8

[9] X. Hu, S.E. Li, Z. Jia, B. Egardt

Energy, 65 (2014), pp. 953-960, 10.1016/j.energy.2013.11.061

URL: http://www.sciencedirect.com/science/article/pii/S0360544213010311

[10] A. Barré, F. Suard, M. Grard, M. Montaru, D. Riu

J. Power Sources, 245 (2014), pp. 846-856

URL: http://dx.doi.org/10.1016/j.jpowsour.2013.07.052

http://www.sciencedirect.com/science/article/pii/S037877531301241X

[11] J. Vetter, P. Novák, M. Wagner, C. Veit, K.-C. Möller, J. Besenhard, M. Winter, M.WohlfahrtMehrens, C. Vogler, A. Hammouche

J. Power Sources, 147 (1-2) (2005), pp. 269-281, 10.1016/j.jpowsour.2005.01.006

URL: http://www.sciencedirect.com/science/article/pii/S0378775305000832

[12] M. Dubarry, C. Truchot, B.Y. Liaw

J. Power Sources, 219 (2012), pp. 204-216, 10.1016/j.jpowsour.2012.07.016

URL: http://www.sciencedirect.com/science/article/pii/S0378775312011330 
[13] H. Xuebing, O. Minggao, L. Languang, L. Jianqiu

Energies, 7 (8) (2014), pp. 4895-4909, 10.3390/en7084895

URL: http://www.mdpi.com/1996-1073/7/8/4895

[14] M. Dubarry, B.Y. Liaw

J. Power Sources, 194 (1) (2009), pp. 541-549, 10.1016/j.jpowsour.2009.05.036

URL: http://www.sciencedirect.com/science/article/pii/S0378775309009604

[15] X. Han, M. Ouyang, L. Lu, J. Li, Y. Zheng, Z. Li

J. Power Sources, 251 (2014), pp. 38-54

URL: http://dx.doi.org/10.1016/j.jpowsour.2013.11.029

http://www.sciencedirect.com/science/article/pii/S0378775313018569

[16] W. Waag, S. Kbitz, D.U. Sauer

Appl. Energy, 102 (2013), pp. 885-897

Special Issue on Advances in sustainable biofuel production and use - \{XIX $\}$ International Symposium on Alcohol Fuels - ISAF, URL:

http://dx.doi.org/10.1016/j.apenergy.2012.09.030

http://www.sciencedirect.com/science/article/pii/S030626191200671X

[17] A. Papoulis. Signal Analysis

McGraw-Hill (1978)

[18] M.B.I. Reaz, M.S. Hussain, F. Mohd-Yasin

Biol. Proced. Online, 8 (December 2006), pp. 11-35

[19] M.S. Crouse, R.D. Nowak, R.G. Baraniuk

IEEE Trans. Signal Process., 46 (4) (1998), pp. 886-902

[20] A.K. Jain, R.C. Dubes. Algorithms for Clustering Data

Prentice-Hall (1988)

[21] C. Lu, L. Tao, F. Fan

J. Power Sources, 261 (2014), pp. 141-147

URL: http://dx.doi.org/10.1016/j.jpowsour.2014.03.058

http://www.sciencedirect.com/science/article/pii/S0378775314003711

[22] J. Aach, G.M. Church, Bioinformatics 17 (6) (2001) 495-508, arXiv: http://bioinformatics.oxfordjournals.org/content/17/6/495.full.pdf + html, http://dx.doi.org/10.10 93/bioinformatics/17.6.495,

URL: http://bioinformatics.oxfordjournals.org/content/17/6/495.abstract.

[23] Z. Bar-Joseph, G. Gerber, D.K. Gifford, T.S. Jaakkola, I. Simon

Proceedings of the Sixth Annual International Conference on Computational Biology, RECOMB

'02, ACM, New York, NY, USA (2002), pp. 39-48, 10.1145/565196.565202

URL: http://doi.acm.org/10.1145/565196.565202

[24] F. Petitjean, C. Kurtz, N. Passat, P. Ganarski

Pattern Recognit. Lett., 33 (13) (2012), pp. 1805-1815, 10.1016/j.patrec.2012.06.009

URL: http://www.sciencedirect.com/science/article/pii/S0167865512001973

[25] H. Sakoe, S. Chiba

Proceedings of the Seventh International Congress on Acoustics, Budapest (1971)

[26] K. Beyer, J. Goldstein, R. Ramakrishnan, U. Shaft 
C. Beeri, P. Buneman (Eds.), Database Theory ICDT'99, Vol. 1540 of Lecture Notes in Computer Science, Springer Berlin Heidelberg (1999), pp. 217-235, 10.1007/3-540-49257-7_15

URL: http://dx.doi.org/10.1007/3-540-49257-7_15

[27] S. Johnson

Psychometrika, 32 (3) (1967), pp. 241-254, 10.1007/BF02289588

URL: http://dx.doi.org/10.1007/BF02289588

[28] V. Vapnik. The Nature of Statistical Learning Theory

Springer-Verlag, New York (1995)

[29] T.S. Furey, N. Cristianini, N. Duffy, D.W. Bednarski, M. Schummer, D. Haussler, Bioinformatics 16 (10) (2000) 906-914,

arXiv: http://bioinformatics.oxfordjournals.org/content/16/10/906.full.pdfthtml, http://dx.doi.org/10.1 093/bioinformatics/16.10.906,

URL: http://bioinformatics.oxfordjournals.org/content/16/10/906.abstract.

[30] S. Tong, D. Koller

J. Mach. Learn. Res., 2 (2002), pp. 45-66, 10.1162/153244302760185243

[31] L.J. Cao, F. Tay

IEEE Trans. Neural Netw., 14 (6) (2003), pp. 1506-1518, 10.1109/TNN.2003.820556

[32] L. Zhang, F. Lin, B. Zhang

Proceedings of the 2001 International Conference on Image Processing, vol. 2 (2001), pp. 721724, 10.1109/ICIP.2001.958595

[33] M.N.H. Shimodaira, K.I. Noma, S. Sagayama

Interspeech (2001), pp. 1841-1844

[34] C.-W. Hsu, C.-J. Lin

IEEE Trans. Neural Netw., 13 (2) (2002), pp. 415-425, 10.1109/72.991427 